\begin{tabular}{c}
\hline \hline Juntendo \\
Research Profiles \\
\hline \hline Juntendo Medical Journal \\
2015. $61(2), 179$
\end{tabular}

\title{
Department of Cell Biology and Neuroscience
}

\section{Principal Investigator: Masato Koike (Professor)}

Our research aims to understand how genetic mutations lead to cellular disorganization and disease, with special emphasis on neurodegenerative diseases related to autophagy, endo-lysosomes, and secretory pathways. Our research team integrates cell and molecular biology, biochemistry, genetics, and light and electron microscopy.

\section{Group Leaders and Research Topics}

\section{1) Masato Koike (Professor)}

We have been analyzing brain tissues from cathepsin D-deficient mice, a model for the most severe type of neuronal ceroid lipofuscinoses (NCLs). NCLs are classified as rare inherited neurodegenerative diseases characterized by the accumulation of mitochondrial proteins in lysosomes. However, taking into account that, in many cases of early-onset familial Parkinson's disease (PD), genes essential for mitophagy are mutated, NCLs and PD may share common etiological features. Indeed, mutations in the ATP13A2 gene are a known cause of Kufor-Rakeb syndrome (KRS), with both PD phenotypes and NCL pathology. We are going to continue genetic study of mouse models with impairment of autophagy and/or lysosomal proteolysis in order to obtain a better understanding of common neurodegenerative diseases.

\section{2) Masato Koike (Professor)}

Currently, the demand for techniques bridging the gap between light and electron microscopy is expanding in the biomedical field. In collaboration with individuals at the Laboratory of Morphology and Image Analysis, one of the biggest and best equipped support facilities in Japanese medical schools, we are establishing a more practical CLEM (correlative light and electron microscopy) method and applying 3D imaging of cells and tissues by focused ion beam/scanning electron microscopy
(FIB/SEM). Furthermore, in collaboration with Prof. Klumperman, a chair of the Department of Cell Biology and Cell Microscopy Core, Medical Center Utrecht, where Prof. Koike worked in 2014 as a visiting professor, we promote advanced Tokuyasu cryosectioning techniques for CLEM.

\section{3) Isei Tanida (Associate Professor)}

We focused on the molecular mechanism in the formation of autophagosomes during autophagy, especially Atg7-mediated ubiqutylation-like reactions. In addition, we are developing tools for monitoring autophagy.

\section{4) Takehiko Sunabori (Assistant Professor)}

We have started to analyze brain-specific conditional knockout (cKO) mouse deficient in p18/LAMTOR1, a late endosome/lysosome-specific anchor protein, for the mTORC1/MAPK signaling pathway.

\section{5) Yu-Shin Sodaka (Assistant Professor)}

We have started to analyze brain- and pancreatic beta cell-specific cKO mouse deficient in Golgi pH regulator (GPHR), an anion channel critical for acidification and functions of the Golgi apparatus.

Publications $\left({ }^{*} \mathrm{Co}-\right.$ first author $)$

1) Nanao $T^{*}$, Koike $M^{*}$, et al: Cellular localization and tissue distribution of endogenous DFCP1 protein. Biomed Res, in press.

2) Asano $T^{*}$, Koike $M^{*}$, et al: Iron induces mitochondrial damage that recruits parkin. Neurosci Lett, 2015; 588C: 29-35.

3) Koike $\mathrm{M}^{*}$, Tanida I ${ }^{*}$, et al: Enrichment of GABARAP relative to LC3 in the axonal initial segments of neurons. PLoS One, 2013; 8: e63568.

4) Koike M, et al: Differences in expression patterns of cathepsin $\mathrm{C} /$ dipeptidyl peptidase I in normal, pathological and aged mouse central nervous system. Eur J Neurosci, 2013; 37: 816-830.

5) Nada $\mathrm{S}^{*}$, Hondo $\mathrm{A}^{*}$, Kasai A*, Koike $\mathrm{M}^{*}$, et al: The novel lipid raft adaptor p18 controls endosome dynamics by anchoring the MEK-ERK pathway to late endosomes. EMBO J, 2009; 28: 477-489. 